\title{
Ratiometric fluorescence sensor based on dithiothreitol modified carbon dots-gold nanoclusters for the sensitive detection of mercury ions in water samples
}

\author{
Wen Liu ${ }^{\mathrm{a}, \mathrm{b}}$, Xiaoyan Wang ${ }^{\mathrm{a}, \mathrm{b}, \mathrm{c}}$, Yunqing Wang ${ }^{\mathrm{b}}$, Jinhua $\mathrm{Li}^{\mathrm{b}}$, Dazhong Shen ${ }^{\mathrm{a}}$, Qi Kang ${ }^{\mathrm{a}, *}$, \\ Lingxin Chen ${ }^{\mathrm{b}, *}$ \\ a College of Chemistry, Chemical Engineering and Materials Science, Collaborative Innovation Center of Functionalized Probes for Chemical Imaging in \\ Universities of Shandong, Key Laboratory of Molecular and Nano Probes, Ministry of Education, Shandong Provincial Key Laboratory of Clean Production of \\ Fine Chemicals, Shandong Normal University, Jinan 250014, China \\ ${ }^{\mathrm{b}}$ Key Laboratory of Coastal Environmental Processes and Ecological Remediation, Research Center for Coastal Environmental Engineering Technology of \\ Shandong Province, Yantai Institute of Coastal Zone Research, Chinese Academy of Sciences, Yantai 264003, China \\ ' School of Pharmacy, Binzhou Medical University, Yantai 264003, China
}

\section{A R T I C L E I N F O}

\section{Article history:}

Received 7 September 2017

Received in revised form 22 January 2018

Accepted 29 January 2018

Available online 1 February 2018

\section{Keywords:}

Mercury ions

Ratiometric fluorescence

Carbon dots-gold nanoclusters

Dithiothreitol

Water samples

\begin{abstract}
A B S T R A C T
Ratiometric fluorescence sensors can provide more accurate analysis of target objects because of their self-calibration function. Traditional methods often use steps of chemical coupling with two different fluorescent materials as the dual emission source, which make the construction process complicated and hard to control. Here, we report a convenient and effective way to construct a ratiometric fluorescence sensor based on dual-emission carbon dots-gold nanoclusters (C-AuNCs) functionalized with dithiothreitol (DTT) for the sensitive detection of mercury ions $\left(\mathrm{Hg}^{2+}\right)$ in water samples. As a type of novel nanoparticle, $\mathrm{C}$-AuNCs are synthesized via microwaving a mixture of gold seeds solution and carbon precursor. In the presence of $\mathrm{Hg}^{2+}$, the free thiol group of DTT would attract them around the surface of $\mathrm{C}$-AuNCs, and owing to the strong $5 \mathrm{~d}^{10}-5 \mathrm{~d}^{10}$ metallophilic interactions between $\mathrm{Hg}^{2+}$ and $\mathrm{Au}^{+}$, the fluorescence emission at $598 \mathrm{~nm}$ is quenched while the emission at $466 \mathrm{~nm}$ remains at a constant intensity. Thus, an obvious color change from orange-red to blue under ultraviolet irradiation can be observed by the naked eye. Moreover, the as-prepared fluorescence sensor has high sensitivity with a detection limit of $8.7 \mathrm{nM}$, and successfully applies to detect $\mathrm{Hg}^{2+}$ in real water samples with satisfactory recoveries at three spiking levels ranging from $95.9-103.5 \%$. The good results imply that the developed DTT/C-AuNCs sensor is conducive to trace $\mathrm{Hg}^{2+}$ determination and possesses the great application potential for water-quality monitoring.
\end{abstract}

(C) 2018 Elsevier B.V. All rights reserved.

\section{Introduction}

Heavy metal ions are one of the most hazardous and widespread contaminants. Mercury(II) ion $\left(\mathrm{Hg}^{2+}\right)$ as a typical heavy metal ion has the feature of non-biodegradable and bio-enrichment, which causes a serious threat to ecological environment and human health. The toxicity of $\mathrm{Hg}^{2+}$ results from its powerful affinity with thiol groups in intracellular proteins and enzymes, leading to the dysfunction of living body [1-3]. Therefore, the United States Environmental Protection Agency (EPA) has defined $10 \mathrm{nM}$ as the maximum allowed limits (MAL) of $\mathrm{Hg}^{2+}$ in qualified drinking water.

\footnotetext{
* Corresponding authors.

E-mail addresses: kangqi9764@163.com (Q. Kang), lxchen@yic.ac.cn (L. Chen).
}

Accordingly, it is necessary to develop reliable and sensitive methods to detect $\mathrm{Hg}^{2+}$ in complex matrices at low concentrations. Conventional analytical techniques mainly include atomic absorption spectroscopy (AAS) [4], inductively coupled plasma-atomic emission spectroscopy (ICP-AES) [5], inductively coupled plasmamass spectrometry (ICPMS) [6], and surface-enhanced Raman scattering (SERS) [7]. However, they commonly require tedious sample preparation, precise equipment and a well-trained operator, which make it difficult to implement in on-site applications. In recent years, fluorescent sensors have emerged for the determination of $\mathrm{Hg}^{2+}$ in biological and environmental systems because of their convenience, rapid response and high sensitivity [8-11]. For example, Li's group synthesized high-yield photoluminescent $\mathrm{N}$-doped carbon nanodots and developed a smartphone application as a platform for the detection of $\mathrm{Hg}^{2+}$ [12].Wang and his 
co-worker developed a Coumarin-based turn-on fluorescent sensor for $\mathrm{Hg}^{2+}$ analysis in neat aqueous solution [13]. Wei and Li utilized DNA-templated silver nanocluster as a fluorescence probe for the sensitive detection of $\mathrm{Hg}^{2+}$ and favorably applied it to real water samples [14].

On the other hand, it is well-known that ratiometric fluorescence sensors have the advantage of avoiding environmental interference and give a more precise measurement attributing to their built-in correction by comparing with two emission intensity ratios rather than using the absolute intensity of a single emission peak [15-17]. Moreover, another unique feature of ratiometric fluorescence analysis is the distinct color change with the variation of analytes dosage under ultraviolet (UV) light. Generally, building dual emission fluorescence sensors often involves taking a substance composited on the surface of another one to form a core-shell construction [18-21]. However, because most nanocomposites are hybrids from different particles, their intrinsic properties may be attacked in a multistep synthesis and sophisticated chemical coupling process. Excitingly, the emergence of carbon dots-gold nanoclusters (C-AuNCs) has broadened our horizon to realize novel dual-fluorescence nanomaterials. Liu's group proposed the synthetic route of C-AuNCs for the first time and successfully applied them to cellular imaging [22]. Unlike the usual C-AuNCs nanocomposites synthesized by combining carbon dots (CDs) with gold nanoclusters (AuNCs), the formation of C-AuNCs only requires microwaving a gold seeds solution containing glucose, which would avoid further chemical conjugation and simplify the experimental steps. In addition, considering the robust metallophilic interaction between $\mathrm{Hg}^{2+}$ and $\mathrm{Au}^{+}$[23-25], C-AuNCs could be an ideal candidate for the ratiometric fluorescence detection of $\mathrm{Hg}^{2+}$.

In the present work, dithiothreitol (DTT) was used to modify the surface of C-AuNCs, which could greatly improve the sensitivity of $\mathrm{Hg}^{2+}$ detection. It should be noticed that DTT plays an important part in the sensing process due to its two thiol groups $(-\mathrm{SH})$ at both ends. Although the thiol group has high affinity for $\mathrm{Hg}^{2+}$, it does not make an impact on $\mathrm{Hg}^{2+}-\mathrm{Au}^{+}$interaction. As previously reported, ethylene diamine tetraacetic acid (EDTA) chelating with $\mathrm{Hg}^{2+}$ had no obvious influence on AuNCs for the detection of $\mathrm{Hg}^{2+}$, which was attributed to the oxidation state of $\mathrm{Hg}^{2+}$ being unchanged [26,27]. Similarly, this explanation can also account for the reason why DTT would not disturb the interaction between $\mathrm{Hg}^{2+}$ and $\mathrm{Au}^{+}$. Therefore, when $\mathrm{Hg}^{2+}$ appears, most can be quickly attracted to the surface of C-AuNCs, followed by the orange-red fluorescence of the AuNCs emission at $598 \mathrm{~nm}$ gradually fading away, whereas the blue fluorescence of $\mathrm{CDs}$ emission at $466 \mathrm{~nm}$ displays a negligible change since it is insensitive to $\mathrm{Hg}^{2+}$. As the two emission intensity ratios vary, the fluorescence color of C-AuNCs changes from orange-red to light blue under a UV lamp, which can be discerned by the naked eye. The constructed ratiometric fluorescence sensor based on DTT modified C-AuNCs, namely DTT/C-AuNCs, was well characterized and its recognition/sensing properties were investigated in detail. The sensor was also applied for the detection of $\mathrm{Hg}^{2+}$ in lake and tap water with satisfactory results. Accordingly, the sensor exhibits excellent sensitivity and selectivity to $\mathrm{Hg}^{2+}$ and provides a convenient platform for visual detection of heavy metal ions in real water samples.

\section{Experimental}

\subsection{Reagents and materials}

Hydrogen tetrachloroaurate trihydrate $\left(\mathrm{HAuCl}_{4} \cdot 3 \mathrm{H}_{2} \mathrm{O}\right)$, glucose, phosphate buffered saline (PBS), and all metal cation salts $\left(\mathrm{Hg}^{2+}\right.$, $\mathrm{Na}^{+}, \mathrm{K}^{+}, \mathrm{Ca}^{2+}, \mathrm{Ba}^{2+}, \mathrm{Mg}^{2+}, \mathrm{Zn}^{2+}, \mathrm{Mn}^{2+}, \mathrm{Ni}^{2+}, \mathrm{Co}^{2+}, \mathrm{Fe}^{3+}, \mathrm{Cd}^{2+}, \mathrm{Pb}^{2+}$,
$\left.\mathrm{Cu}^{2+}\right)$ and sodium anion salts $\left(\mathrm{I}^{-}, \mathrm{F}^{-}, \mathrm{HSO}_{4}^{-}, \mathrm{CN}^{-}, \mathrm{CH}_{3} \mathrm{COO}^{-}\right.$, $\mathrm{H}_{2} \mathrm{PO}_{4}^{-}$) were purchased from Sinopharm Chemical Reagent Co. Ltd. (Shanghai, China). Reduced glutathione (GSH) and ethylene diamine tetraacetic acid (EDTA) were supplied by Aladdin Industrial Co. (Shanghai, China). Dithiothreitol (DTT) was obtained from Sigma-Aldrich Trading Company Ltd. (Shanghai, China). Pure water purification system was purchased from Pall Co. (New York, USA) and ultrapure water with a resistivity of $18.2 \mathrm{M} \Omega$ was used for all experiments. All reagents used were of analytical grade without any further purification.

\subsection{Instrumentation}

Fluorescence spectra were measured with a Fluoromax-4 Spectrofluorometer (Horiba Scientific, Japan). The morphological evaluation was examined by a transmission electron microscope (TEM) (JEOL, model JEM-1230, Japan) operated at $100 \mathrm{kV}$. UV-vis absorption spectra were recorded on a NanoDrop 2000/2000C spectrophotometer (Thermo Fisher Scientific, USA). Fourier transform infrared (FT-IR) analyses were carried out by a FT-IR spectrometer (Thermo Fisher Scientific, Model Nicolet iS10, USA). Atomic fluorescence analysis was measured by an atomic fluorescence spectrophotometer (AFS) (Jitian Instruments, Model AFS-930, China).

\subsection{Synthesis of $C-A u N C S$}

All glass wares were soaked with aqua regia $\left(\mathrm{HCl}: \mathrm{HNO}_{3}=3: 1\right)$ and rinsed thoroughly with ultrapure water before use. C-AuNCs were prepared by the microwave-assistant seed growth method according to previous report with minor modification [22,28]. Typically, $2 \mathrm{~mL}$ of $50 \mathrm{mM}$ freshly prepared $\mathrm{HAuCl}_{4}$ aqueous solution was injected into a round-bottomed flask, and then the solution was diluted by ultrapure water up to $48.5 \mathrm{~mL}$ at room temperature. After mixing uniformly, $1.5 \mathrm{~mL}$ of $100 \mathrm{mM} \mathrm{GSH}$ was added to the above solution and heated to $70^{\circ} \mathrm{C}$ for $2 \mathrm{~h}$ to form gold seeds. Next, $15 \mathrm{mg}$ of glucose was mixed with $5 \mathrm{~mL}$ of fresh gold seeds solution followed by microwaving the mixture for $8 \mathrm{~min}$. Finally, the product was dissolved again with ultrapure water and centrifuged $(12,000 \mathrm{rpm}, 10 \mathrm{~min})$ to remove agglomerated particles. In this way, the dual emission C-AuNCs were purified, which were stored at $4{ }^{\circ} \mathrm{C}$ for further use.

\subsection{Synthesis of DTT functionalized C-AuNCS}

Briefly, $20 \mu \mathrm{L}$ of $4 \mathrm{mM}$ DTT and $2 \mathrm{~mL}$ of C-AuNCs aqueous solution were added to $8 \mathrm{~mL}$ of ultrapure water. Then, the mixture was stirred for $12 \mathrm{~h}$ in the dark [29]. The product was centrifuged $(12,000 \mathrm{rpm}, 15 \mathrm{~min})$ to remove superfluous amounts of DTT. At last, the green-yellow solution of DTT functionalized C-AuNCs marked as DTT/C-AuNCs was obtained.

\subsection{Ratiometric fluorescence detection of mercury ions}

In a centrifuge tube, $200 \mu \mathrm{L}$ of DTT/C-AuNCs aqueous solution was diluted with $290 \mu \mathrm{L}$ of PBS buffer $(10 \mathrm{mM}, \mathrm{pH} 7.0)$, and then $10 \mu \mathrm{L}$ of $\mathrm{Hg}^{2+}$ solution with a known concentration was added to the above DTT/C-AuNCs sensor solution. After 10 min of incubation, the well-prepared working solution was transferred to a quartz cuvette for the fluorescence measurements. The final concentration of DTT/C-AuNCs used in the fluorescence test was $0.20 \mathrm{mg} / \mathrm{mL}$. All fluorescence (FL) intensities were obtained in the same conditions: both excitation and emission slit widths were set as $8 \mathrm{~nm}$, and the value of excitation wavelength was $380 \mathrm{~nm}$. 


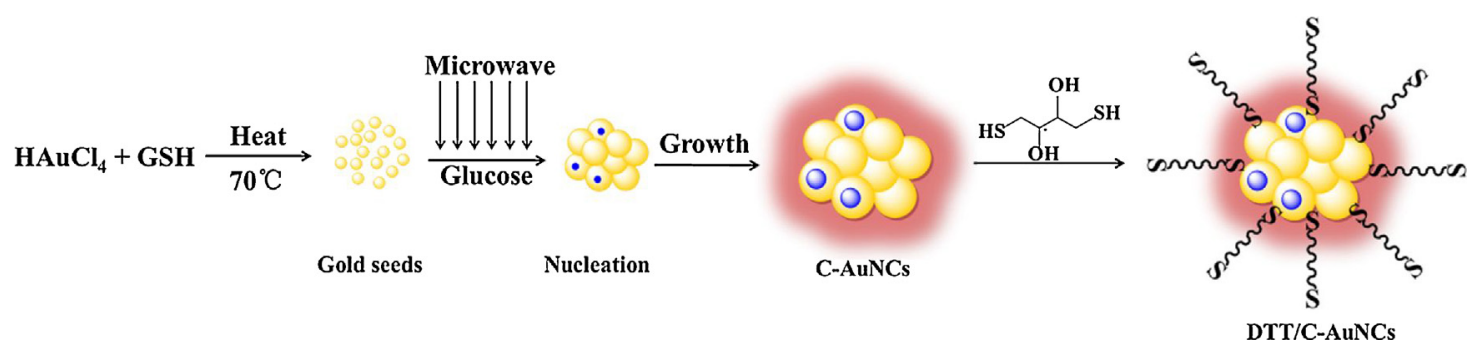

Fig. 1. Schematic illustration of the synthetic routes of DTT/C-AuNCs.

\subsection{Selectivity and interference experiment}

To investigate the selectivity of DTT/C-AuNCs, the fluorescent responses to other ions such as metallic ions $\left(\mathrm{Na}^{+}, \mathrm{K}^{+}, \mathrm{Ca}^{2+}, \mathrm{Mg}^{2+}\right.$, $\left.\mathrm{Co}^{2+}, \mathrm{Ni}^{2+}, \mathrm{Ba}^{2+}, \mathrm{Mn}^{2+}, \mathrm{Zn}^{2+}, \mathrm{Fe}^{3+}, \mathrm{Cu}^{2+}, \mathrm{Cd}^{2+}, \mathrm{Pb}^{2+}\right)$ and anions $\left(\mathrm{I}^{-}\right.$, $\mathrm{F}^{-}, \mathrm{HSO}_{4}^{-}, \mathrm{CN}^{-}, \mathrm{CH}_{3} \mathrm{COO}^{-}, \mathrm{H}_{2} \mathrm{PO}_{4}^{-}$) were examined under the same conditions as that of $\mathrm{Hg}^{2+}$ mentioned above. The concentrations of $\mathrm{Hg}^{2+}$ and other ions were all $500 \mathrm{nM}$. For the interference study, $20 \mu \mathrm{M} \mathrm{Na}^{+}, \mathrm{K}^{+}, \mathrm{Ca}^{2+}, \mathrm{Mg}^{2+}, \mathrm{Co}^{2+}, \mathrm{Ni}^{2+}, \mathrm{Ba}^{2+}, \mathrm{Mn}^{2+}, \mathrm{Zn}^{2+}, \mathrm{Fe}^{3+}$, $\mathrm{I}^{-}, \mathrm{F}^{-}, \mathrm{HSO}_{4}{ }^{-}, \mathrm{CN}^{-}, \mathrm{CH}_{3} \mathrm{COO}^{-}$, and $\mathrm{H}_{2} \mathrm{PO}_{4}^{-}$and $5 \mu \mathrm{M} \mathrm{Cu}^{2+}, \mathrm{Cd}^{2+}$, and $\mathrm{Pb}^{2+}$ were mixed with the DTT/C-AuNCs sensor solution. In the presence of interfering ions, $500 \mathrm{nM} \mathrm{Hg}^{2+}$ was then added to the sensor solution and the fluorescence spectra were recorded again.

\subsection{Analysis of water samples}

Lake water and tap water were employed to demonstrate the practicality of the DTT/C-AuNCs sensor for $\mathrm{Hg}^{2+}$ detection in aqueous matrices. The lake water sample was taken from San Yuan Lake (Yantai, China), and tap water sample was collected from our laboratory after water was allowed to flow for $5 \mathrm{~min}$. The real water samples were primitively filtered with a $0.45 \mu \mathrm{m}$ pore size membrane to remove the suspended particles. After 100 -fold dilution, the prepared water samples were spiked with $\mathrm{Hg}^{2+}$ standard solution at $0.1,0.5$, and $1 \mu \mathrm{M}$. Then, the fluorescence spectra were measured after incubating for $10 \mathrm{~min}$.

\section{Results and discussion}

\subsection{Preparation and detection principle of DTT/C-AuNCs}

C-AuNCs were the foundation for the construction of the DTT/C-AuNCs ratiometric fluorescence sensor. Therefore, C-AuNCs were preferentially prepared and characterized. According to a previous work, C-AuNCs were synthesized by the microwave-assisted method, and the detailed process is illustrated in Fig. 1. As seen, gold seeds were first obtained via the reduction of $\mathrm{HAuCl}_{4}$ with GSH as a stabilizer. Then, the gold seeds solution containing glucose was subjected to microwave irradiation to form C-AuNCs. Finally, the C-AuNCs were dissolved in ultrapure water for further modification by DTT. In addition, because the amounts of glucose could control the fluorescence intensity of CDs, different glucose dosages were tested in the formation of C-AuNCs. Ultimately, $15 \mathrm{mg}$ of glucose was selected as the optimal dosage for the experiment to obtain our desired dual-emission peak pattern of C-AuNCs.

Fig. 2A displays the fluorescence spectra of AuNCs (curve a), C-AuNCs (curve b) and CDs (curve c). Under microwave irradiation, the mixture of glucose $(15 \mathrm{mg}$ ) and gold seeds $(5 \mathrm{~mL})$ formed C-AuNCs within $8 \mathrm{~min}$. In the same case as mentioned above, independent gold seeds $(5 \mathrm{~mL}$ ) could form AuNCs. However, when only $15 \mathrm{mg}$ of glucose was dissolved in $5 \mathrm{~mL}$ of pure water and the solution was microwaved for $8 \mathrm{~min}$, the obtained products did not show fluorescence emission. The generation of CDs needs a high concentration of glucose solution (such as $0.1 \mathrm{~g} \mathrm{~mL}^{-1}$ ) by the microwave-assisted method. That is, $\mathrm{C}$-AuNCs is different from the composite of CDs and AuNCs. The possible mechanism for the formation of C-AuNCs has been discussed [22], which includes the two processes of nucleation and growth. The nucleation rate of CDs is lower than that of AuNCs, while the growth rate of CDs is higher. Carbon will have a priority to nucleate on the surface of gold seeds for generating C-AuNCs. The TEM images of AuNCs and C-AuNCs are shown in Fig. 2B. From the images, we can deduce that $\mathrm{C}-\mathrm{AuNCs}$ have an average size of approximately $3 \mathrm{~nm}$, which is similar to that of AuNCs. This further proves that C-AuNCs are nanoparticles in terms of particle size.

The synthesis principle of DTT functionalized C-AuNCs is based on the strong covalent interaction of the thiol group with AuNCs [30]. After a period of stirring, a large number of collisions would guarantee that the DTT is effectively connected to the surface of C-AuNCs (Fig. 1). The FT-IR spectra are used to characterize the functional groups of C-AuNCs and DTT/C AuNCs. As shown in Fig. 3, C-AuNCs and DTT/CAuNCs have mostly the same characteristic peaks. The peak at $3428 \mathrm{~cm}^{-1}$ corresponds to the stretching vibration of $-\mathrm{OH}$, and the peak at $1646 \mathrm{~cm}^{-1}$ confirms the existence of $-\mathrm{COOH}$. The $-\mathrm{C}-\mathrm{N}$ and $\mathrm{S}-\mathrm{Au}$ bands are observed at 1402 and $1076 \mathrm{~cm}^{-1}$, respectively [31]. The emergence of $\mathrm{S}-\mathrm{Au}$ bands in the C-AuNCs FT - IR spectra can be attributed to GSH as the reducing reagent involved in the formation of gold seeds. A weak peak at $2487 \mathrm{~cm}^{-1}$ appearing in the FT - IR spectra of DTT/C-AuNCs is assigned to the characteristic vibrations of - $\mathrm{SH}$ [32], which manifests that only one end of $-\mathrm{SH}$ is anchored on the surface of $\mathrm{C}$-AuNCs while the other end of $-\mathrm{SH}$ is free in the process of DTT modification.

As a ligand, DTT plays an essential role in the detection of $\mathrm{Hg}^{2+}$ because of its unique structure. Taking advantage of the covalent bond between - SH and Au, DTT can easily attach to C-AuNCs, while the other end of $-\mathrm{SH}$ is responsible for grabbing $\mathrm{Hg}^{2+}$ [30]. In the presence of $\mathrm{Hg}^{2+}$, the free $-\mathrm{SH}$ exclusively attracts $\mathrm{Hg}^{2+}$ through the $\mathrm{S}-\mathrm{Hg}$ band [33], resulting in the fluorescence emission at $598 \mathrm{~nm}$ being sharply quenched. The sensing mechanism could be attributed to the powerful dispersion forces between closed-shell metal atoms, leading to the formation of a strong metallophilic bond between the $\mathrm{d}^{10}$ centers of $\mathrm{Au}^{+}\left(5 \mathrm{~d}^{10}\right)$ and $\mathrm{Hg}^{2+}\left(5 \mathrm{~d}^{10}\right)$ [3,23,34-37]. The established metallophilic bond would efficiently quench the fluorescence of AuNCs. Therefore, as the concentration of $\mathrm{Hg}^{2+}$ increased, the orange-red fluorescence of AuNCs decreased, whereas the blue fluorescence of $\mathrm{CDs}$ as a reference signal was almost constant. Subsequently, the DTT/C-AuNCs based ratiometric fluorescence sensor displayed a continuous color change from orange-red to blue with the addition of $\mathrm{Hg}^{2+}$, which can be visualized under ultraviolet irradiation, as shown in Fig. 4. Although the UV absorption spectrum of AuNCs and the fluorescence spectrum of CDs had a small overlap region (Fig. S1), there did not exist an energy transfer between AuNCs and CDs, since the fluorescence intensity of CDs was almost unchanged. 
A

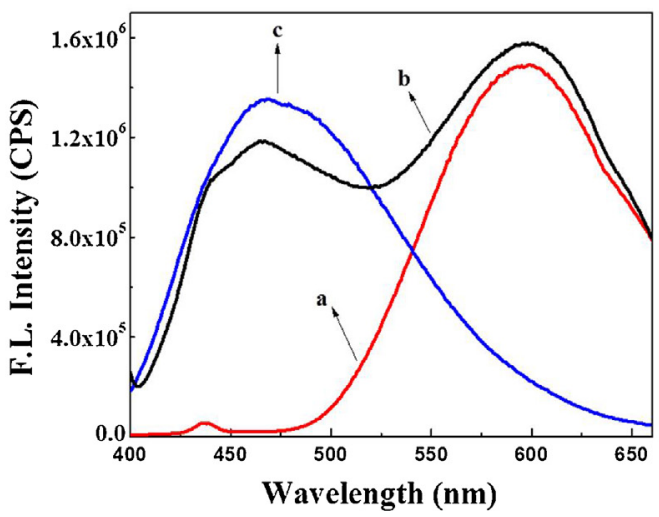

B
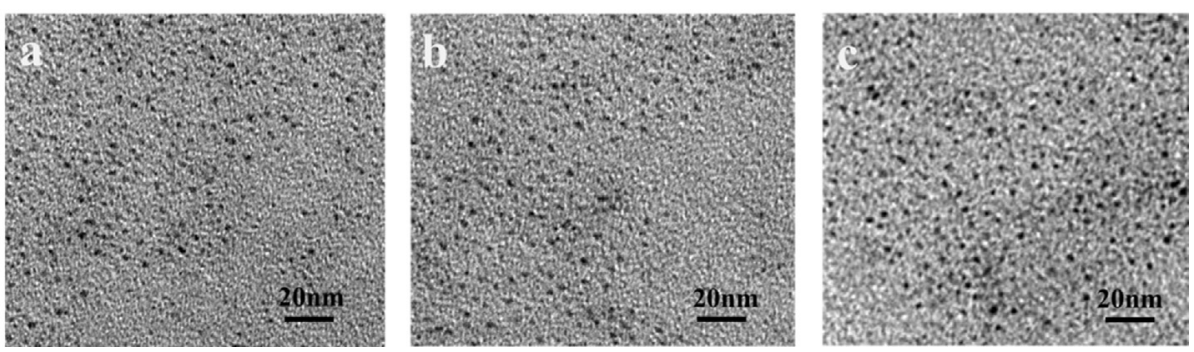

Fig. 2. (A) Fluorescence emission spectra $\left(\lambda_{\mathrm{ex}}=380 \mathrm{~nm}\right.$ ) of (a) AuNCs, (b) C-AuNCs, and (c) CDs. (B) TEM images of (a) AuNCs, (b) C-AuNCs and (c) DTT/C-AuNCs.

A

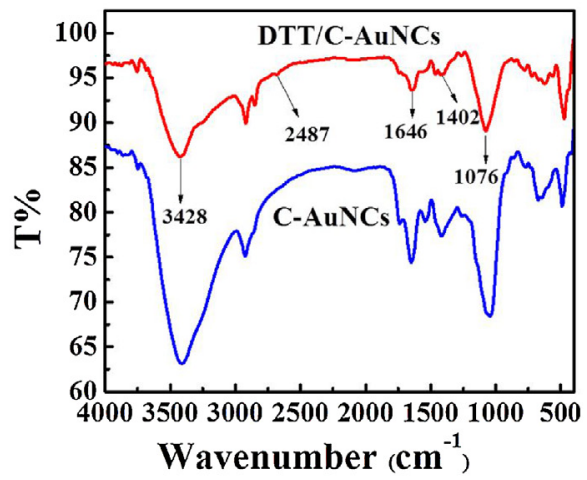

B

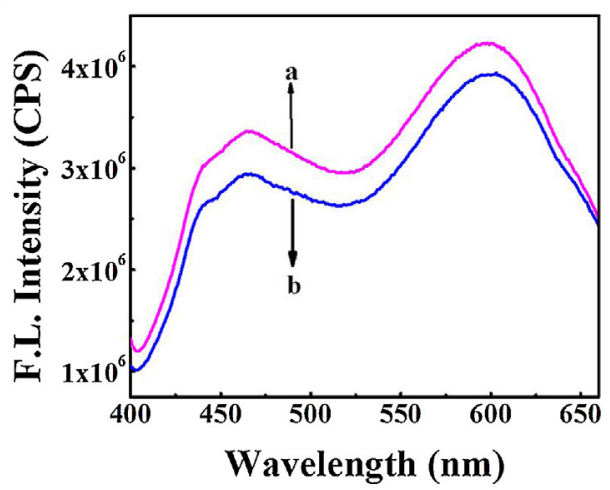

Fig. 3. (A) The FT - IR spectra of C-AuNCs and DTT/C-AuNCs. (B) The fluorescence spectra ( $\lambda$ ex $=380 \mathrm{~nm}$ ) of C-AuNCs (a) before and (b) after DTT modification.

\subsection{Morphological structure and optical properties of DTT/C-AuNCS}

The morphological structures of C-AuNCs and DTT/C-AuNCs were characterized by TEM. From Fig. 2B, we can see that the particles size of C-AuNCs did not change after DTT modification, which indicated that DTT would not cause aggregation of C-AuNCs. In addition, the fluorescence spectra of C-AuNCs and DTT/C-AuNCs were also recorded (Fig. 3B). As seen, the emission peak at $598 \mathrm{~nm}$ of DTT/C-AuNCs had a small redshift compared to that of C-AuNCs, but the relative fluorescence intensity of the two peaks remained unchanged. From the above, we can conclude that DTT as a modifier was friendly to C-AuNCs.

The quenching amount, defined as $\left(\mathrm{F}_{0}-\mathrm{F}\right) / \mathrm{F}_{0}$ [38], was chosen as a criterion for evaluating the effect of pH. From Fig. S2, it was found that the quenching amount sequentially increased below $\mathrm{pH} 7.0$ but clearly decreased as the $\mathrm{pH}$ value ranged from 7.5 to
9.0. The phenomenon may be attributed to that $\mathrm{Hg}^{2+}$ can transform into $\mathrm{Hg}(\mathrm{OH})_{2}$ in an alkaline medium, which caused a decrease in the actual concentrations of $\mathrm{Hg}^{2+}$. Moreover, owing to the $\mathrm{pK}_{\mathrm{a}}$ of the thiol group being 8.3 [39], DTT would be oxidized when the $\mathrm{pH}$ value is higher than 8.0. Considering the above reasons and the practical application of the DTT/C-AuNCs sensor for environment water, we chose $\mathrm{pH} 7.0$ in PBS buffer as the optimized condition for further experiments. Meanwhile, the response time of the fluorescence sensor was also investigated by monitoring its fluorescence intensity decrease as the sensor solution reacted with $500 \mathrm{nM} \mathrm{Hg}^{2+}$. Fig. S3 displays that the fluorescence intensity at $598 \mathrm{~nm}$ declined rapidly and reached stabilization in $10 \mathrm{~min}$. The results suggested that $\mathrm{Hg}^{2+}$ would easily come into contact with the surface of C-AuNCs and had a strong interaction with $\mathrm{Au}^{+}$. Therefore, $10 \mathrm{~min}$ of response time was enough for the complete reaction between $\mathrm{Hg}^{2+}$ and DTT/C-AuNCs. 

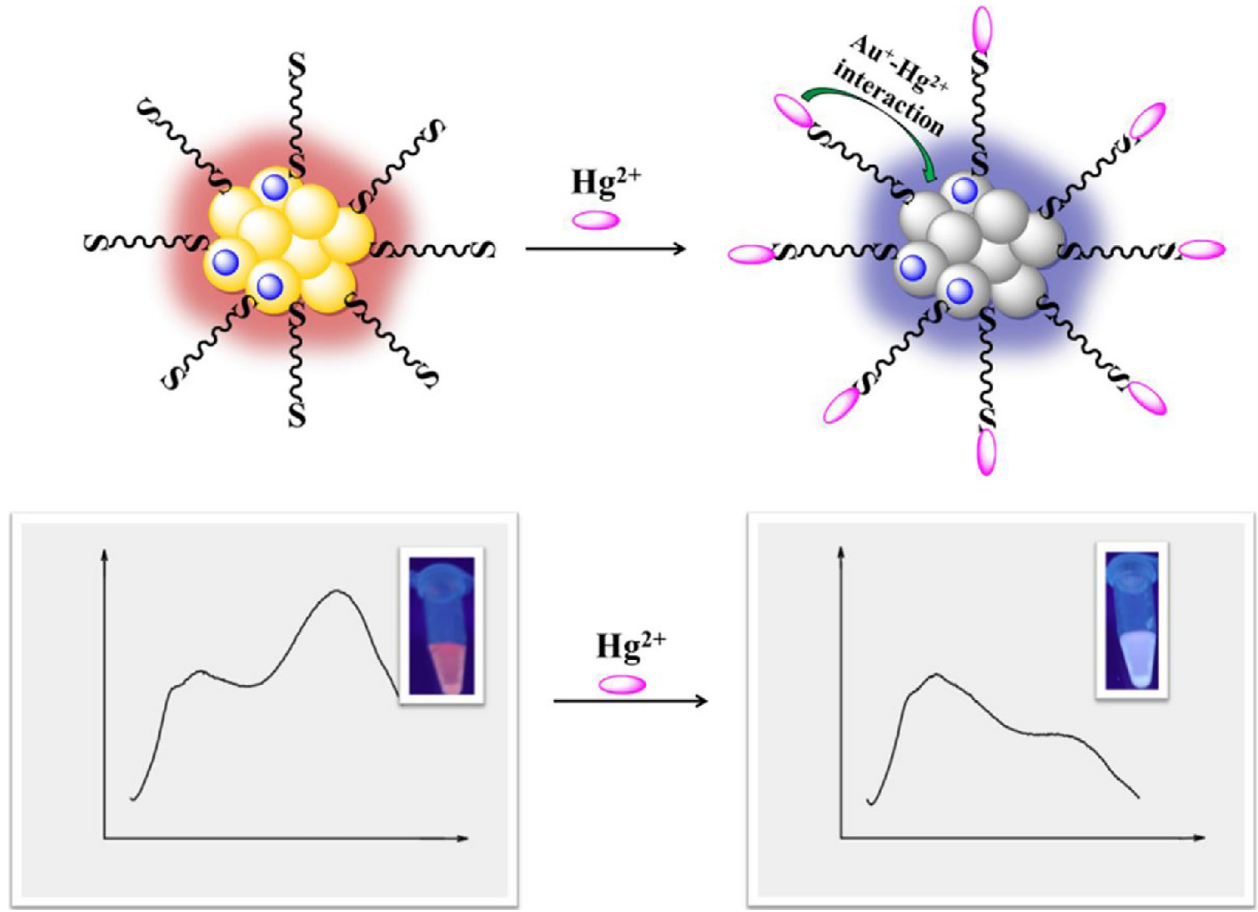

Fig. 4. Schematic illustration of the proposed quenching mechanism of the senor for $\mathrm{Hg}^{2+}$ detection.

\subsection{Sensitivity and selectivity of the sensor to $\mathrm{Hg}^{2+}$}

The ability of the DTT/C-AuNCs sensor for the quantitative analysis of $\mathrm{Hg}^{2+}$ was evaluated by recording the fluorescence emission intensity after the addition of different concentrations of $\mathrm{Hg}^{2+}(0$, 0.05, 0.1, 0.2, 0.3, 0.4, 0.5, 0.6, 0.7, 0.8, 0.9 and $1 \mu \mathrm{M}$ ) (Fig. 5A). An excellent linear relationship between the intensity ratio and $\mathrm{Hg}^{2+}$ concentrations was presented with $\mathrm{R}^{2}=0.9981$ (Fig. 5B). The limit of detection (LOD), defined by the equation LOD $=3 \sigma / s$, where $\sigma$ is the standard deviation of blank signal and $s$ represents the slope of the calibration curve, was calculated to be $8.7 \mathrm{nM}$. The value was lesser than the MAL for $\mathrm{Hg}^{2+}$ in drinking water, i.e., $10 \mathrm{nM}$ regulated by the EPA. These results ensured the validity of the prepared ratiometric fluorescence sensor for $\mathrm{Hg}^{2+}$ detection.

On the other hand, high selectivity is a pivotal assessment index for an excellent fluorescent sensor, which stands for the ability of the sensor to distinguish between the target analyte and interferent. The selectivity of DTT/C-AuNCs was demonstrated by measuring the fluorescence emission ratios $\left(\mathrm{I}_{598} / \mathrm{I}_{466}\right)$ (Fig. $5 \mathrm{C}$ and D) in the presence of $\mathrm{Hg}^{2+}$ and multiple interfering ions, including metal ions $\left(\mathrm{Na}^{+}, \mathrm{K}^{+}, \mathrm{Ca}^{2+}, \mathrm{Ba}^{2+}, \mathrm{Mg}^{2+}, \mathrm{Zn}^{2+}, \mathrm{Mn}^{2+}, \mathrm{Ni}^{+}, \mathrm{Co}^{2+}\right.$, $\left.\mathrm{Fe}^{3+}, \mathrm{Cd}^{2+}, \mathrm{Pb}^{2+}, \mathrm{Cu}^{2+}\right)$ and anions $\left(\mathrm{Cl}^{-}, \mathrm{Br}^{-}, \mathrm{I}^{-}, \mathrm{F}^{-}, \mathrm{HSO}_{4}^{-}, \mathrm{CH}_{3} \mathrm{COO}^{-}\right.$, $\mathrm{H}_{2} \mathrm{PO}_{4}^{-}$). It was found that $\mathrm{Cu}^{2+}$ would cause a disturbance for $\mathrm{Hg}^{2+}$ detection. As shown in Fig. S4A, approximately $30 \%$ of fluorescence intensity at $598 \mathrm{~nm}$ (curve d) was quenched by $500 \mathrm{nM} \mathrm{Hg}^{2+}$, while the quenching amount of $500 \mathrm{nM} \mathrm{Cu}^{2+}$ could reach $14 \%$ (curve c), which nearly equaled to half the quenched ability of $\mathrm{Hg}^{2+}$. To solve this problem, EDTA was employed as a masking agent to eliminate the interference of $\mathrm{Cu}^{2+}$, since EDTA is a popular ligand toward most cations (with stability constant $\log \mathrm{K}\left(\mathrm{Cu}^{2+}\right.$-EDTA $)=18.80$ ). Control experiments have been performed to verify that EDTA is useful for masking $\mathrm{Cu}^{2+}$ and would not hinder the detection of $\mathrm{Hg}^{2+}$. When $0.1 \mathrm{mM}$ EDTA was introduced into the working solution containing $500 \mathrm{nM} \mathrm{Cu}{ }^{2+}, 99 \%$ of the original fluorescence intensity at $598 \mathrm{~nm}$ (curve b) was obtained which was almost identical to that of the mixture of DTT/C-AuNCs and EDTA (curve a) (Fig. S4A). Moreover, the fluorescence intensity of DTT/C-AuNCs react- ing with $500 \mathrm{nM} \mathrm{Hg}^{2+}$ (curve d) remained unchanged after the addition of $0.1 \mathrm{mM}$ EDTA (curve e) (Fig. S4A). In addition, we also investigated the masking ability of EDTA towards $5 \mu \mathrm{M} \mathrm{Cu}^{2+}$ in the condition of coexisting with $500 \mathrm{nM} \mathrm{Hg}^{2+}$. Fig. S4 B shows that $5 \mu \mathrm{MCu}^{2+}$ can sharply quench the fluorescence intensity at $598 \mathrm{~nm}$, which was approximately equivalent to the quenching efficiency of $1 \mu \mathrm{M}$ of $\mathrm{Hg}^{2+}$. Fortunately, in the presence of EDTA, the fluorescence intensity curve of $5 \mu \mathrm{M} \mathrm{Cu}^{2+}$ mixed with $500 \mathrm{nM} \mathrm{Hg}^{2+}$ coincided with that of only $500 \mathrm{nM} \mathrm{Hg}^{2+}$ existing in the working solution, which means that EDTA can effectively chelate with $\mathrm{Cu}^{2+}$ to eliminate interference. Moreover, the EDTA would not have any influence on DTT/C-AuNCs because the fluorescence spectrum remained unchanged after the addition of EDTA to the blank working solution (blank working solution means only DTT/C-AuNCs in PBS buffer at $\mathrm{pH}$ 7.0). Although $\mathrm{Hg}^{2+}$ also binds with EDTA $\left(\log \left(\mathrm{Hg}^{2+}-\mathrm{EDTA}\right)=21.7\right)$, the complexation did not alter mercury's oxidation state, and the quenching effect of DTT/C-AuNCs still remained normal [27]. In addition, the thiol group of DTT may have stronger affinity with $\mathrm{Hg}^{2+}$, so $\mathrm{Hg}^{2+}$ recognition was not disturbed. As seen from Figs. 5C and S5, after $\mathrm{Cu}^{2+}$ was sheltered, the ratiometric fluorescence sensor possessed a good selectivity to $\mathrm{Hg}^{2+}$ and the fluorescence color changes were not obvious in the presence of other ions. For the interference study, the fluorescence intensity ratio $\left(\mathrm{I}_{598} / \mathrm{I}_{466}\right)$ was not influenced by adding other ions at relatively high concentrations $\left(20 \mu \mathrm{M} \mathrm{Na}{ }^{+}, \mathrm{K}^{+}, \mathrm{Ca}^{2+}, \mathrm{Ba}^{2+}, \mathrm{Mg}^{2+}, \mathrm{Zn}^{2+}\right.$, $\mathrm{Mn}^{2+}, \mathrm{Ni}^{2+}, \mathrm{Co}^{2+}, \mathrm{Fe}^{3+}, \mathrm{I}^{-}, \mathrm{F}^{-}, \mathrm{HSO}_{4}^{-}, \mathrm{CN}^{-}, \mathrm{CH}_{3} \mathrm{COO}^{-}, \mathrm{H}_{2} \mathrm{PO}_{4}^{-} ; 5 \mu \mathrm{M}$ $\mathrm{Cd}^{2+}, \mathrm{Pb}^{2+}, \mathrm{Cu}^{2+}\left(\mathrm{Cu}^{2+}\right.$ is sheltered by EDTA $\left.)\right)$ to the working solution containing $500 \mathrm{nM} \mathrm{Hg}^{2+}$, as shown in Fig. S6. The results indicated that the ratiometric fluorescence sensor exhibited high selectivity and recognition specificity for $\mathrm{Hg}^{2+}$ in contrast to other ions.

\subsection{Practical application of the ratiometric fluorescence sensor to water samples}

To further assess the practical application of the proposed method to real samples, the lake water and tap water were spiked with working solutions containing different concentrations of $\mathrm{Hg}^{2+}$ 
A

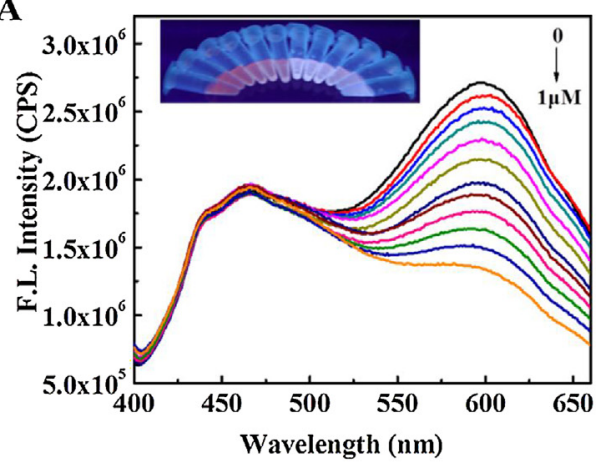

C

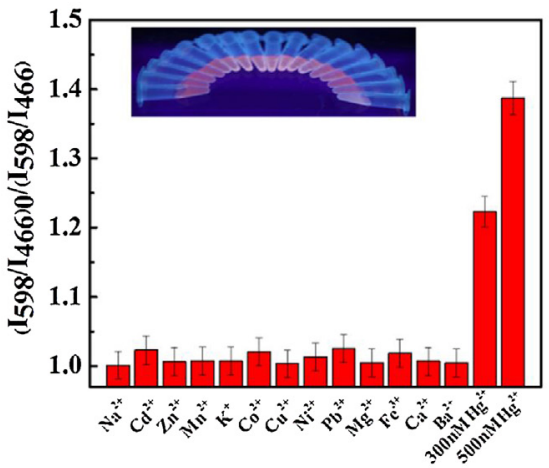

B

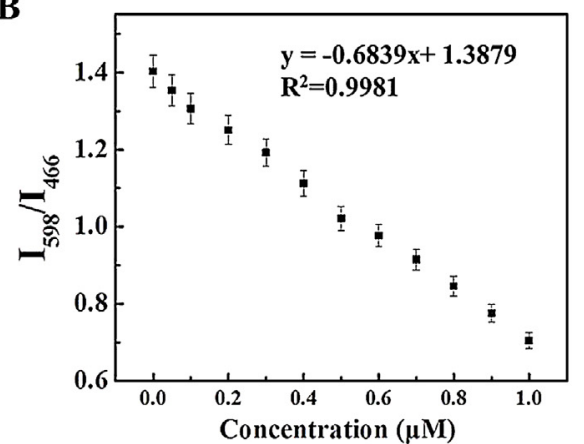

D

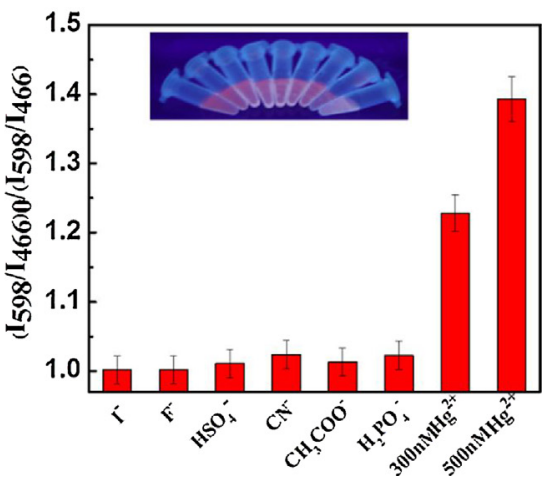

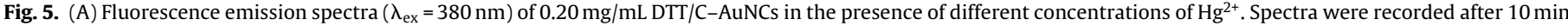

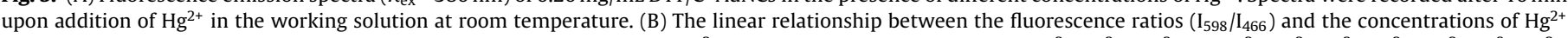

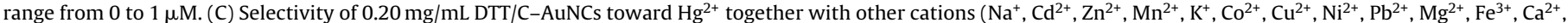

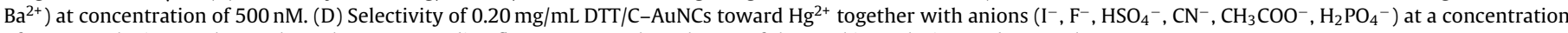
of $500 \mathrm{nM}$. The insert photos show the corresponding fluorescence colors change of the working solution under a UV lamp.

Table 1

Results of spiked recoveries and RSDs (\%; $\mathrm{n}=3$ ) for the detection of $\mathrm{Hg}^{2+}$ in lake water and tap water samples with the DTT/C-AuNCs sensor.

\begin{tabular}{|c|c|c|c|c|c|}
\hline \multirow[t]{2}{*}{ Sample } & \multirow{2}{*}{$\begin{array}{l}\text { Spiked } \\
(\mu \mathrm{M})\end{array}$} & \multicolumn{2}{|c|}{ DTT/C-AuNCs sensor } & \multicolumn{2}{|l|}{ AFS } \\
\hline & & Recovery $^{\mathrm{a}}(\%)$ & RSD (\%) & Recovery $^{\mathrm{a}}(\%)$ & RSD (\%) \\
\hline \multirow[t]{3}{*}{ Lake water } & 0.1 & 95.9 & 2.8 & 101.3 & 1.6 \\
\hline & 0.5 & 101.0 & 3.1 & 104.2 & 2.0 \\
\hline & 1.0 & 103.5 & 3.9 & 109.7 & 2.5 \\
\hline \multirow[t]{3}{*}{ Tap water } & 0.1 & 97.2 & 2.4 & 103.0 & 1.9 \\
\hline & 0.5 & 98.9 & 1.8 & 97.8 & 2.3 \\
\hline & 1.0 & 101.5 & 3.3 & 106.7 & 2.7 \\
\hline
\end{tabular}

a Average value from three individual experiments.

and then tested. The averaged recovery was acquired with the relative standard deviations (RSD) by measuring three triplicates for each concentration. The data of the spiking experiment are listed in Table 1. As shown, satisfactory recoveries were attained at $95.9-103.5 \%$ for the spiked lake water samples and $97.2-101.5 \%$ for the spiked tap water samples, respectively. The value of RSD below $4 \%$ demonstrated the reliability and accuracy of the ratiometric fluorescence sensor. In addition, the results were comparable to that obtained by AFS, namely, 101.3-109.7\% for lake water samples and $97.8-106.7 \%$ for tap water samples. These good results confirmed that the DTT/C-AuNCs sensor was suitable for $\mathrm{Hg}^{2+}$ detection in drinkable and environmental water samples, possessing great potential for the analysis and monitoring of $\mathrm{Hg}^{2+}$ in practice.

\subsection{Method performance comparison}

The performance of the developed DTT/C-AuNCs ratiometric fluorescence sensor for the detection of $\mathrm{Hg}^{2+}$ was compared with other different AuNCs-based fluorescence detection approaches, as listed in Table S1. Some researchers have reported the use of different proteins or enzymes as the stabilizer for the synthesis of AuNCs for improving the detection effect of $\mathrm{Hg}^{2+}$ [40-43], but these methods cannot show a color change as the concentration of $\mathrm{Hg}^{2+}$ varies. In our work, we constructed a ratiometric fluorescence sensor, employing AuNCs as a response signal to discern $\mathrm{Hg}^{2+}$, which could visually detect $\mathrm{Hg}^{2+}$ according to an obvious color change from orange-red to blue. In addition, there are other ratiometric fluorescence sensors for detecting $\mathrm{Hg}^{2+}$. For example, Yan's group reported a method of utilizing CDs and AuNCs to form a nanocomposite for sensitively detecting $\mathrm{Hg}^{2+}$ [44]. However, the synthesis process of AuNCs was time-consuming and the CDs needed a high temperature $\left(200^{\circ} \mathrm{C}\right)$ to form. In addition, Wang et al. designed a dual-emitting fluorescent chemosensor based on fluorescence resonance energy transfer with blue fluorescent poly(arylene ether nitrile) acting as the energy donor and redemitting AuNCs as the acceptor for detecting $\mathrm{Hg}^{2+}$ [45]. Similarly, this strategy also involved tedious synthetic routes. Although in our work, the obtained detection limit was comparable or lower than those mentioned ratiometric fluorescence detection methods, the CAuNCs synthesized by the microwave-assisted method dramatically simplified the preparation process and avoided complicated chemical conjugation.

\section{Conclusions}

In summary, we introduced a simple and feasible method to construct a ratiometric fluorescence sensor for the sensitive recognition of $\mathrm{Hg}^{2+}$ with the help of a novel multifunction nanoparticle C-AuNCs modified by DTT. The fluorescence system showed a lower detection limit of $8.7 \mathrm{nM}$ with a wide linearity range from 
$50 \mathrm{nM}$ to $1000 \mathrm{nM}$ and was successfully applied to detection of $\mathrm{Hg}^{2+}$ in real water samples. Based on the specific strong interactions between $\mathrm{Hg}^{2+}$ and $\mathrm{Au}^{+}$, the fluorescence intensity at $598 \mathrm{~nm}$ of AuNCs was quenched in the presence of $\mathrm{Hg}^{2+}$, while the emission peak at $466 \mathrm{~nm}$ of CDs was almost invariable as the reference signal, accompanied by an observable fluorescence color change under UV light. In this work, DTT played the role of attracting $\mathrm{Hg}^{2+}$ appearing on the surface of the AuNCs to increase the effective contact with $\mathrm{Au}^{+}$, to enhance the recognition ability. Considering the high accuracy and excellent sensitivity/selectivity, the developed sensor has great potential for practical application in water pollution monitoring.

On the other hand, the emergence of multifunction dualemission nanoparticles no longer restricts the ratiometric fluorescence method to be composited by two different fluorescence materials, so it is important to explore the preparation process and application. Moreover, for fluorescence sensors, more attention should be paid to explain the possible sensing principle, which is beneficial for exploiting their application area. Finally, we expect that the development of dual emission fluorescence nanoparticles would strongly promote the prosperity of ratiometric sensors in concerned-target analysis.

\section{Acknowledgments}

This work was financially supported by the National Natural Science Foundation of China (21575080, 21275091, 21275158, 21477160 ), the National Defense Science and Technology Innovation Project of Chinese Academy of Sciences (CXJJ-16M254), and the Scientific Research Foundation for the Returned Overseas Chinese Scholars, State Education Ministry.

\section{Appendix A. Supplementary data}

Supplementary data associated with this article can be found, in the online version, at https://doi.org/10.1016/j.snb.2018.01.222.

\section{References}

[1] D.T. Quang, J.S. Kim, Fluoro-and chromogenic chemodosimeters for heavy metal ion detection in solution and biospecimens, Chem. Rev. 110 (2010) 6280-6301.

[2] C. Zhang, Z.G. Yu, G.M. Zeng, M. Jiang, Z.Z. Yang, F. Cui, M.Y. Zhu, L.Q. Shen, L. $\mathrm{Hu}$, Effects of sediment geochemical properties on heavy metal bioavailability, Environ. Int. 73 (2014) 270-281.

[3] L. Shang, L.X. Yang, F. Stockmar, R. Popescu, V. Trouillet, M. Bruns, D. Gerthsen, G.U. Nienhaus, Nanoscale 4 (2012) 4155-4160.

[4] H. Erxleben, J. Ruzicka, Atomic absorption spectroscopy for mercury, automated by sequential injection and miniaturized in lab-on-valve system, Anal. Chem. 77 (2005) 5124-5128.

[5] Z.L. Zhu, G.C.Y. Chan, S.J. Ray, X.R. Zhang, G.M. Hieftje, Use of a solution cathode glow discharge for cold vapor generation of mercury with determination by ICP-atomic emission spectrometry, Anal. Chem. 80 (2008) $7043-7050$.

[6] H.T. Chen, J.G. Chen, X.Z. Jin, D.Y. Wei, Determination of trace mercury species by high performance liquid chromatography-inductively coupled plasma mass spectrometry after cloud point extraction, J. Hazard. Mater. 172 (2009) $1282-1287$.

[7] Y.J. Ding, S.S. Wang, J.H. Li, L.X. Chen, Nanomaterial-based optical sensors for mercury ions, Trends Anal. Chem. 82 (2016) 175-190.

[8] S. Yoon, A.E. Albers, A.P. Wong, C.J. Chang, Screening mercury levels in fish with a selective fluorescent chemosensor, J. Am. Chem. Soc. 127 (2005) 16030-16031.

[9] K.P. Carter, A.M. Young, A.E. Palmer, Fluorescent sensors for measuring metal pons in living systems, Chem. Rev. 114 (2014) 4564-4601.

[10] W.B. Lu, X.Y. Qin, S. Liu, G.H. Chang, Y.W. Zhang, Y.L. Luo, A.M. Asiri, A.O. Al-Youbi, X.P. Sun, Economical, green synthesis of fluorescent carbon nanoparticles and their use as probes for sensitive and selective detection of mercury (II) ions, Anal. Chem. 84 (2012) 5351-5357.

[11] J. Liu, X.L. Ren, X.W. Meng, Z. Fang, F.Q. Tang, Sensitive and selective detection of $\mathrm{Hg} 2+$ and $\mathrm{Cu} 2+$ ions by fluorescent Ag nanoclusters synthesized via a hydrothermal method, Nano 5 (2013) 10022-10028

[12] L. Wang, B.Q. Li, F. Xu, X.Y. Shi, D.M. Feng, D.Q. Wei, Y. Li, Y.J. Feng, Y.M. Wang, D.C. Jia, Y. Zhou, High-yield synthesis of strong photoluminescent N-doped carbon nanodots derived from hydrosoluble chitosan for mercury ion sensing via smartphone APP, Biosens. Bioelectron. 79 (2016) 1-8.

[13] C.J. Wu, J.B. Wang, J.J. Shen, C. Bi, H.W. Zhou, Coumarin-based Hg2+ fluorescent probe: synthesis and turn-on fluorescence detection in neat aqueous solution, Sens. Actuators B 243 (2017) 678-683.

[14] C.Y. Li, C.Y. Wei, DNA-templated silver nanocluster as a label-free fluorescent probe for the highly sensitive and selective detection of mercury ions, Sens. Actuators B 242 (2017) 563-568.

[15] X.Y. Wang, J.L. Yu, X.Q. Wu, J.Q. Fu, Q. Kang, D.Z. Shen, J.H. Li, L.X. Chen, A molecular imprinting-based turn-on Ratiometric fluorescence sensor for highly selective and sensitive detection of 24 -dichlorophenoxyacetic acid (2. 4-D), Biosens. Bioelectron. 81 (2016) 438-444.

[16] K. Zhang, T. Yu, F. Liu, M.T. Sun, H. Yu, B.H. Liu, Z.P. Zhang, H. Jiang, S.H. Wang, Selective fluorescence turn-on and ratiometric detection of organophosphate using dual-emitting Mn-doped ZnS nanocrystal probe, Anal. Chem. 86 (2014) $11727-11733$

[17] P. Zhao, K.Y. He, Y.T. Han, Z. Zhang, M.Z. Yu, H.H. Wang, Y. Huang, Z. Nie, S.Z. Yao, Near-infrared dual-emission quantum dots?gold nanoclusters nanohybrid via co-template synthesis for ratiometric fluorescent detection and bioimaging of ascorbic acid in vitro and in vivo, Anal. Chem. 87 (2015) 9998-10005.

[18] C. Dai, C.X. Yang, X.P. Yan, Ratiometric fluorescent detection of phosphate in aqueous solution based on near infrared fluorescent silver nanoclusters/metal?organic shell composite, Anal. Chem. 87 (2015) $11455-11459$.

[19] A.W. Zhu, Q. Qu, X.L. Shao, B. Kong, Y. Tian, Carbon-dot-based dual-emission nanohybrid produces a ratiometric fluorescent sensor for in vivo imaging of cellular copper ions, Angew. Chem. Int. Ed. 124 (2012) 7297-7301.

[20] P. Wu, C.Y. Xu, X.D. Hou, J.J. Xu, H.Y. Chen, Dual-emitting quantum dot nanohybrid for imaging of latent fingerprints: simultaneous identification of individuals and traffic light-type visualization of TNT, Chem. Sci. 6 (2015) 4445-4450.

[21] X.J. Liu, N. Zhang, T. Bing, D.H. Shangguan, Carbon dots based dual-emission silica nanoparticles as a ratiometric nanosensor for Cu2+, Anal. Chem. 86 (2014) 2289-2296

[22] L.Y. Zhang, D.H. Wang, H.W. Huang, L.F. Liu, Y. Zhou, X.D. Xia, K.Q. Deng, X.Y. Liu, ACS Appl. Mater. Interfaces 8 (2016) 6646-6655.

[23] J.P. Xie, Y.G. Zheng, J.Y. Ying, Highly selective and ultrasensitive detection of $\mathrm{Hg} 2+$ based on fluorescence quenching of Au nanoclusters by $\mathrm{Hg} 2+-\mathrm{Au}+$ interactions, Chem. Commun. 46 (2010) 961-963.

[24] Y.Q. Cai, L. Yan, G.Y. Liu, H.Y. Yuan, D. Xiao, In-situ synthesis of fluorescent gold nanoclusters with electrospun fibrous membrane and application on $\mathrm{Hg}$ (II) sensing, Biosens. Bioelectron. 41 (2013) 875-879.

[25] Y. Zhang, J.J. Jiang, M. Li, P.F. Gao, L.H. Shi, G.M. Zhang, C. Dong, S.M. Shuang, Bright far-red/near-infrared gold nanoclusters for highly selective and ultra-sensitive detection of Hg2+, Sens. Actuators B 238 (2017) 683-692.

[26] S. Bothra, Y. Upadhyay, R. Kumara, S.K. Ashok Kumar, S.K. Sahoo, Chemically modified cellulose strips with pyridoxal conjugated red fluorescent gold nanoclusters for nanomolar detection of mercuric ions, Biosens. Bioelectron. 90 (2017) 329-335.

[27] D.Y. Cao, J. Fan, J.R. Qiu, Y.F. Tu, J.L. Yan, Masking method for improving selectivity of gold nanoclusters in fluorescence determination of mercury and copperions, Biosens. Bioelectron. 42 (2013) 47-50.

[28] H.Z. Lu, S.F. Xu, Visualizing BPA by molecularly imprinted ratiometric fluorescence sensor based on dual emission nanoparticles, Biosens. Bioelectron. 92 (2017) 147-153.

[29] Y.J. Zhou, X.Y. Huang, C. Liu, R.L. Zhang, X.L. Gu, G.J. Guan, C.L. Jiang, L.Y. Zhang, S.H. Du, B.H. Liu, M.Y. Han, Z.P. Zhang, Color-multiplexing-based fluorescent test paper: dosage-sensitive visualization of arsenic (III) with discernable scale as low as 5 ppb, Anal. Chem. 88 (2016) 6105-6109.

[30] A. Senthamizhan, A. Celebioglu, B. Balusamy, T. Uyar, Immobilization of gold nanoclusters inside porous electrospun fibers for selective detection of $\mathrm{Cu}$ (II): a strategic approach to shielding pristine performance, Sci. Rep. 5 (2015) 15608

[31] X.H. Xu, X. Qi, X.Q. Wang, X.Y. Wang, Q. Wang, H. Yang, Y.C. Fu, S.Z. Yao, Highly efficient enzyme immobilization by nanocomposites of metal organic coordination polymers and carbon nanotubes for electrochemical biosensing, Electrochem. Commun. 79 (2017) 18-22.

[32] A. Gupta, A. Chaudhary, P. Mehta, C. Dwivedi, S. Khan, N.C. Verma, C.K. Nandi, Nitrogen-doped, thiol-functionalized carbon dots for ultrasensitive $\mathrm{Hg}$ (II) detection, Chem. Commun. 51 (2015) 10750-10753.

[33] Y. Zhao, H.Q. Liu, F. Chen, M. Bai, J.W. Zhao, Y.X. Zhao, Trifunctional molecular beacon-mediated quadratic amplification for highly sensitive and rapid detection of mercury (II) ion with tunable dynamic range, Biosens. Bioelectron. 86 (2016) 892-898.

[34] M. Kim, T.J. Taylor, F.P. Gabbai, Hg(II) … Pd(II) metallophilic interactions, J. Am. Chem. Soc. 130 (2008) 6332-6333.

[35] Y.H. Lin, W.L. Tseng, Ultrasensitive sensing of $\mathrm{Hg} 2+$ and $\mathrm{CH} 3 \mathrm{Hg}+$ based on the fluorescence quenching of lysozyme type VI-stabilized gold nanoclusters, Anal. Chem. 82 (2010) 9194-9200.

[36] X. Yuan, T.J. Yeow, Q.B. Zhang, J.Y. Lee, J.P. Xie, Highly luminescent Ag+ nanoclusters for Hg2+ ion detection, Nanoscale 4 (2012) 1968-1971.

[37] P. Pyykko, Theoretical chemistry of gold. III, Chem. Soc. Rev. 37 (2008) 1967-1997 
[38] S.F. Xu, H.Z. Lu, One-pot synthesis of mesoporous structured ratiometric fluorescence molecularly imprinted sensor for highly sensitive detection of melamine from milk samples, Biosens. Bioelectron. 73 (2015) 160-166.

[39] N. Bi, Y.H. Chen, H.B. Oi, X. Zheng, Y. Chen, X. Liao, H.O. Zhang, Y. Tian, Spectrophotometric determination of mercury (II) ion using gold nanorod as probe, Sens. Actuators B 166 (2012) 766-771.

40] Y. Zhang, M.F. Yan, J.J. Jiang, P.F. Gao, G.M. Zhang, M.F. Choi-Martin, C. Dong, S.M. Shuang, Highly selective and sensitive nanoprobes for $\mathrm{Hg}$ (II) ions based on photoluminescent gold nanoclusters, Sens. Actuators B 235 (2016 386-393.

[41] Y.Y. Qiao, Y. Zhang, C.H. Zhang, L.H. Shi, G.M. Zhang, S.M. Shuang, C.H. Dong, M. Ma, Water-soluble gold nanoclusters-based fluorescence probe for highly selective and sensitive detection of $\mathrm{Hg} 2+$, Sens. Actuators B 224 (2016) 458-464.

[42] N.Y. Hsu, Y.W. Lin, Microwave-assisted synthesis of bovine serum albumin-gold nanoclusters and their fluorescence-quenched sensing of Hg2 ions, New J. Chem. 40 (2016) 1155-1161.

[43] H.C. Chang, Y.F. Chang, N.C. Fan, J.A. Ho, Facile preparation of high-quantum-yield gold nanoclusters: application to probing mercuric ions and biothiols, ACS Appl. Mater. Interfaces 6 (2014) 18824-18831.

[44] Y.H. Yan, H. Yu, K. Zhang, M.T. Sun, Y.J. Zhang, X.K. Wang, S.H. Wang, Dual-emissive nanohybrid of carbon dots and gold nanoclusters for sensitive determination of mercuric ions, Nano Res. 9 (7) (2016) 2088-2096.

[45] P. Wang, L.Y. Zhao, H.G. Shou, J.Y. Wang, P.L. Zheng, K. Jia, X.B. Liu, Dual-emitting fluorescent chemosensor based on resonance energy transfer from poly (arylene ether nitrile) to gold nanoclusters for mercury detection, Sens. Actuators B 230 (2016) 337-344.

\section{Biographies}

Wen Liu received her BS degree from School of Chemistry, Chemistry and Chemical Engineering, Qufu Normal University, in 2015. She is studying for her master degree in School of Chemistry, Chemical Engineering and Materials Science, Shan dong Normal University, Joint-Educated at Yantai Institute of Coastal Zone Research, Chinese Academy of Sciences. Her current research interest is molecularly imprinted polymers based chemical sensors for environmental analysis.

Xiaoyan Wang received her PhD in Shandong Normal University, Joint-Educated at Yantai Institute of Coastal Zone Research, Chinese Academy of Sciences, in 2017.
Her current research interests focus on the preparation \& application of molecularly imprinted polymers in chromatographic separation and chemical sensors for analysis of typical pollutants.

Yunqing Wang received his $\mathrm{PhD}$ in Pharmaceutical Analysis from China Pharmaceutical University, Nanjing, China, in 2009. During the same year, he joined the Laboratory of Environmental Microanalysis and Monitoring of Prof. Lingxin Chen at the Yantai Institute of Coastal Zone Research, Chinese Academy of Sciences, as a research assistant. In 2013, he was promoted to an associate professor. His current research interests focus on the development of fluorescence and SERS based nanosensors and the applications in bioimaging.

Jinhua Li received her PhD in Analytical Chemistry from the Department of Chemistry of Hong Kong Baptist University, Hong Kong, in 2009. In the same year, she joined in Yantai Institute of Coastal Zone Research, Chinese Academy of Sciences, as an assistant professor. In 2015, she became an associate professor. Her current research interest focuses on the preparation of novel molecularly imprinted polymers and applications to sample pretreatment and chemo/biosensors.

Dazhong Shen is a professor in School of Chemistry, Chemical Engineering and Materials Science, Shandong Normal University. He received his MSc and PhD degrees in analytical chemistry from Hunan University. His research interests include chemical sensors and biosensors.

Qi Kang is a professor in School of Chemistry, Chemical Engineering and Materials Science, Shandong Normal University. She received her MSc and PhD degrees in analytical chemistry from Shandong University. Her research interests include chemical sensors and biosensors.

Lingxin Chen has been a professor at Yantai Institute of Coastal Zone Research, Chinese Academy of Sciences, since 2009. He obtained his Ph.D. in analytical chemistry at Dalian Institute of Chemical Physics, Chinese Academy of Sciences, in 2003. During 2004-2009, he worked at Department of Chemistry, Tsinghua University, and Department of Applied Chemistry, Hanyang University, respectively. His research interests include the studies of novel properties of materials such as functionalized nanoparticles \& functional probe molecules for developing nanoscale biochemica analysis methods and molecular imprinting-based sample pretreatment technology. He has published 3 academic books, 200 research papers and topical reviews. 